\title{
A Co-Condensation Model for In-Flight Synthesis of Metal-Carbide Nanoparticles in Thermal Plasma Jet
}

\author{
A. Vorobev, O. Zikanov, and P. Mohanty
}

(Submitted June 11, 2008; in revised form July 28, 2008)

\begin{abstract}
We present a theoretical analysis of the formation, growth, and transport of two-component nanoparticles in thermal plasma jet. The approach of the aerosol science and the idea of multicomponent co-condensation are employed for the analysis. The processes of homogeneous nucleation, heterogeneous growth, and coagulations due to Brownian collisions are considered in combination with the convective and diffusive transport of particles and the reacting gases within an axisymmetric domain. As a particular example, the authors study multicomponent co-condensation of metal-carbide nanoparticles from various precursors in a DC plasma gun operating in an argon atmosphere.
\end{abstract}

Keywords Brownian collisions, heterogeneous condensation, homogeneous nucleation, nanoparticles, numerical modeling, refractory coating, thermal plasma spraying

\section{Introduction}

Metal carbides such as $\mathrm{TaC}, \mathrm{SiC}, \mathrm{TiC}$ are used in a wide range of industrial applications due to their excellent high temperature properties as well as chemical/wear resistance. The majority of these carbide powders are produced by carbothermic reduction. Alternatively, thermal plasma spraying is an excellent process for the preparation of metal carbide nanopowders from liquid/gaseous precursors due to its unique characteristics such as high temperature $(>10,000 \mathrm{~K})$, high chemical activity, and rapid quenching $\left(\sim 10^{6-7} \mathrm{~K} / \mathrm{s}\right)$. The use of thermal plasma spraying technique for production of nanopowders has been experimentally explored by several investigators (e.g., Ref 1-4). Several theoretical analyses also have focused on the synthesis of nanoparticles in different plasma reactors (Ref 1, 5-11). Most of the theoretical work devoted to the synthesis of nanoparticles is, however,

This article is an invited paper selected from presentations at the 2008 International Thermal Spray Conference and has been expanded from the original presentation. It is simultaneously published in Thermal Spray Crossing Borders, Proceedings of the 2008 International Thermal Spray Conference, Maastricht, The Netherlands, June 2-4, 2008, Basil R. Marple, Margaret M. Hyland, Yuk-Chiu Lau, Chang-Jiu Li, Rogerio S. Lima, and Ghislain Montavon, Ed., ASM International, Materials Park, OH, 2008.

A. Vorobev, University of Southampton, Southampton, UK; and O. Zikanov and P. Mohanty, University of Michigan-Dearborn, Dearborn, MI. Contact e-mail: A.Vorobev@soton.ac.uk. focused on the nucleation and growth of particles consisting of one component.

Recently, the authors developed an approach for modeling the in-flight synthesis of nanoparticles based on the concept of multicomponent co-condensation and descriptive tools of the aerosol science (Ref 12). Synthesis of TaC nanoparticles in atmospheric condition was considered. Practical applicability of the open atmosphere synthesis is diminished by the oxidation problem (Ref 2). Because of that, in the present paper, the authors extend the analysis to a promising configuration of an argon-filled chamber. Furthermore, the model is applied to examine the synthesis of other practically interesting metal-carbide systems.

Saturation vapor pressures of the condensing materials play an important role in the co-condensation process. In this paper, the authors consider metals that possess lower saturation vapor pressure (e.g., Si and Ti) as well as higher saturation vapor pressure (Ta and $\mathrm{W}$ ) compared to that of carbon. The nucleation and condensation sequence based on their relative saturation vapor pressures and phase diagrams are discussed.

\section{Coating System and Operational Parameters}

The system under consideration has certain specific features since the parameters of the DC plasma gun installed in the laboratory at the University of MichiganDearborn, are used for simulations. However, the general modeling approach to the particle synthesis can be extended to other systems based on the same principal mechanism, namely, fast quenching leading to homogeneous nucleation and subsequent heterogeneous condensation.

The coating system uses a DC plasma gun operating at 38-40 V and $600 \mathrm{~A}$. Pure argon is injected into the plasma gun at the rate of $1.68 \times 10^{-3} \mathrm{~kg} \mathrm{~s}^{-1}$. The plasma gun operates in a chamber filled with argon. Suction is applied 
to maintain constant argon pressure approximately equal to atmospheric pressure in the chamber. Measurements near the chamber walls show temperature of about $300 \mathrm{~K}$.

The formation of nanoparticles is initiated by injection of precursors (such as liquid tantalum ethoxide $\mathrm{Ta}\left(\mathrm{OC}_{2} \mathrm{H}_{5}\right)_{5}$ used for production of $\mathrm{TaC}$ nanoparticles (Ref 2)) into the nozzle. The actual details of injection are unimportant, since it can be assumed that the plasma jet is sufficiently hot to completely evaporate and chemically decompose the precursor and dissociate its atoms. As a result, it is assumed that monoatomic gases of $\mathrm{Ta}$ and $\mathrm{C}$ are formed before the jet exists from the nozzle. The only limiting assumption made in the following is that the precursor loading is low, so that evaporation does not significantly affect the argon flow.

\section{Numerical Model of Gas Flow}

The electromagnetic, thermodynamic, and gas dynamic processes governing the flow within a thermal plasma coating device are extremely complex. A complete model should, in principle, include description of discharge arc, turbulent energy and mass transport in a magnetohydrodynamic flow of real plasma, processes of ionization and deionization, etc. Such models, especially when realistic motions of discharge arc are considered, are unfeasible at today's level of physical understanding and computational capabilities. Even the most advanced models, for example (Ref 13), where dynamic simulations of the flow induced by a torch operating in the takeover mode were conducted, achieved only qualitative agreement with experiments. Since the focus of this investigation is on the formation of nanoparticles rather than on the arc and jet behavior the

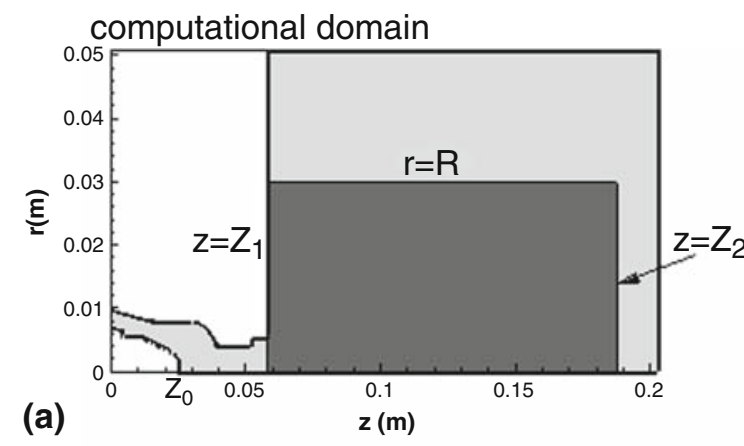

temperature, $\mathrm{K}$

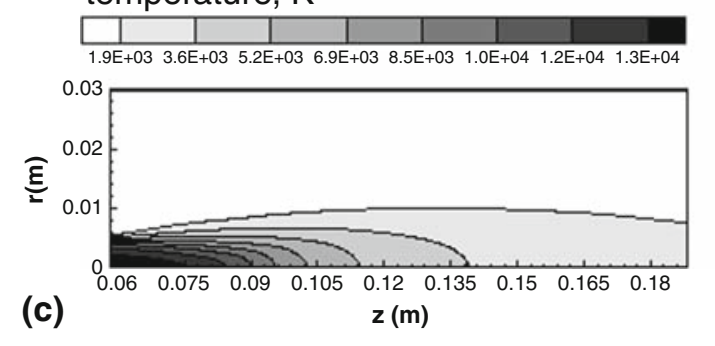

authors obtain the needed properties of the flow by using a simple hydrodynamic model correlated with experimental data. Similar approach was taken in other studies of nanoparticle synthesis (for example, Ref $1,6,8$ ).

The Navier-Stokes equations for a compressible, viscous, and heat-conducting ideal argon gas are solved numerically using the CFD software Fluent. Mean (ensemble averaged) steady-state flow is calculated. The effect of turbulent fluctuations on the mean fields is taken into account via the turbulent eddy viscosity and heat conduction coefficients modeled by the realizable $k-\varepsilon$ turbulence model (Ref 14). The thermodynamic and molecular transport coefficients of argon are computed as functions of temperature following the formulas provided in Ref 15 and 16. The numerical scheme used by Fluent is based on a finite-volume discretization and is of the second order of accuracy. To identify the gird providing acceptably small numerical errors, the authors have conducted simulations with different degrees of grid refinement and clustering in the areas of strong flow gradients.

The computational domain is shown in Fig. 1(a). Axial symmetry is assumed. In accordance with the focus on the synthesis of nanoparticles rather than on the actual coating process, the target wall, which is typically located at the distance of $10-15 \mathrm{~cm}$ from the nozzle, is removed in the model. The chamber walls are also replaced by the free outlet boundaries located far from the nozzle and maintained at constant pressure $p=p_{\text {atm }}$ and temperature $T=300 \mathrm{~K}$. This simplification is supported by the measurements of actual pressure and temperature conditions and justified by the fact that the particle formation occurs in a zone close to the nozzle and at significant distance to the walls. Throughout the entire domain outside the nozzle, the gas pressure is found to be close to

\section{axial velocity, $\mathrm{m} / \mathrm{s}$}
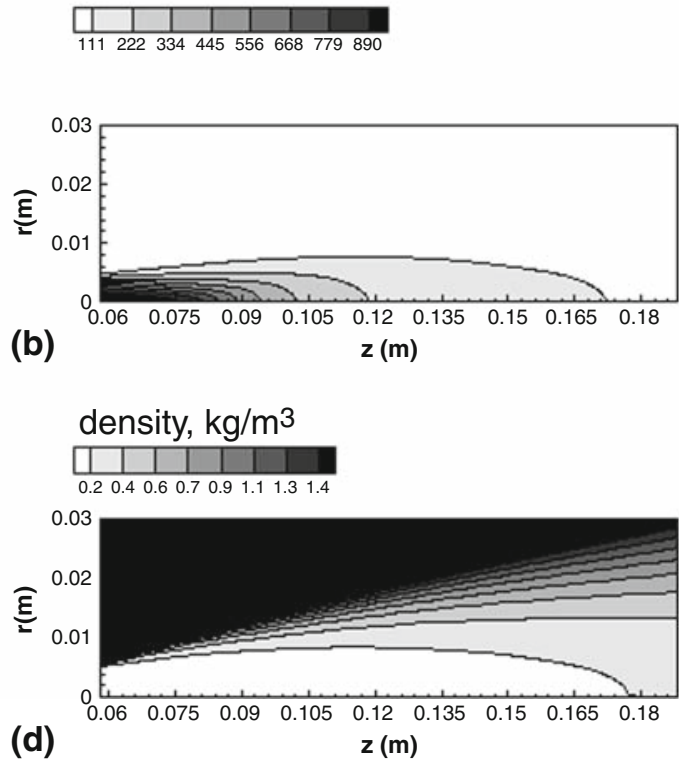

Fig. 1 Results of the simulations of the gas flow. Full computational domain (light gray) and the domain where the evolution of particles is studied (dark gray) (a). Distributions of axial velocity (b), temperature (c), and gas density (d) 
atmospheric. The inlet boundary conditions include the mass flux of $1.68 \times 10^{-3} \mathrm{~kg} \mathrm{~s}^{-1}$ of pure argon at temperature of $300 \mathrm{~K}$ and pressure of $1.5 p_{\text {atm }}$

The effect of arc discharge is imitated by the energy input from a heat source located within the plasma gun. This approach, albeit being a drastic simplification of the real flow physics, can be justified by the fact that the nanoparticle formation occurs entirely outside the nozzle. It can be assumed that the parameters of the jet flow in that area are primarily determined by the rate of energy generation within the nozzle and, as an approximation, neglect the details of the energy generation. The power of the heat source is chosen as $16.2 \mathrm{~kW}$. This is small compared with the typical powers at which the DC plasma reactors operate $(22.8-24 \mathrm{~kW}$ for the setup in the authors laboratory). The difference accounts for the losses not taken into account directly in the model. The energy generation rate is distributed within the nozzle interior with the density: $E=E_{0} \exp \left(-2\left(s / s_{0}\right)^{5}\right)$, where $E_{0}=1.35 \times 10^{10} \mathrm{~J} \mathrm{~m}^{-3}, s$ is the distance from the tip of the cathode $\left(z=z_{0}, r=0\right)$ (see Fig. 1a), $z_{0}=2.5 \mathrm{~cm}$, and $s_{0}=z_{0} / 3$.

To account for the energy losses due to cooling of the anode, the convective boundary conditions with the heat transfer coefficient $10^{5} \mathrm{~W} \mathrm{~m}^{-2} \mathrm{~K}^{-1}$ are imposed at its walls. The cathode boundary temperature is constant in time and varies from $300 \mathrm{~K}$ at the inlet to $1000 \mathrm{~K}$ at the cathode tip, according to the following profile: $T=300+700 \exp \left(-2\left(s / s_{0}\right)^{5}\right)$. It should be understood that such temperature boundary conditions represent only an attempt of approximation needed in the absence of reliable experimental or computational data. The possible effect of inaccuracy in the thermal boundary conditions on the temperature and velocity of the jet by varying the parameters, such as the temperature of the cathode surface, heat transfer coefficient for the anode wall, value of the inlet pressure, is investigated. No significant effect was found for plausible (not very large) variations.

Yet another simplifying assumption used in this model was needed because of the difficulty of modeling the effect of the precursor droplets and the heat of their evaporation on the gas flow. Although feasible in principle, accurate modeling of this effect is impossible in this case, since essential thermodynamic properties of liquid precursors (e.g., tantalum ethoxide) are largely unknown for considered temperature ranges. The stages of chemical decomposition, which is likely to include hundreds of chemical reactions, are also difficult to predict. For these reasons the authors chose to limit the cases under consideration to those with low loading, i.e., with the mass flow rate of the precursor much smaller than that of the argon gas.

The results of the numerical calculations for the case chosen for further analysis are shown in Fig. 1. The jet of hot argon is emitted into a chamber filled with cooled argon gas. The turbulent mixing provides a rapid fall in the gas temperature. The rate of the temperature fall is, however, smaller compared with the results obtained for jet emitted into the air atmosphere (Ref 12$)$.

\section{Theoretical and Numerical Model of Formation and Growth of Nanoparticles}

The key elements of the analysis of the particle synthesis are the model of particle formation via the multicomponent co-condensation description using the particle distribution function, and the approximate solution based on the assumption of nearly monodisperse distribution. Only a brief discussion is given here. For a detailed discussion, the reader is referred to the authors' earlier paper (Ref 12).

The nanoparticle formation is modeled within the computational domain marked by the dark gray area in Fig. 1(a). As mentioned above, it is assumed that the liquid precursor molecules are completely dissociated within the nozzle so concentrations of monoatomic gases forming the particles (e.g., Ta and C) can be prescribed at the inlet into the marked zone. Other chemical elements resulting from the precursor dissociation are disregarded in the following analysis. Their existence is partially accounted for by taking the initial concentration of the carbon vapor to be less than the amount of carbon produced by precursor dissociation. This reflects the fact that part of carbon is bounded in, e.g., $\mathrm{CO}$ gas that passively leaves the system. This simplification is inevitable, since analysis of the entire set of chemical reactions expected to occur (more than 100 as shown in Ref 6) is very difficult to conduct for multidimensional spatially variable distributions of reagent concentrations, pressure, and temperature. Further difficulty is due to the lack of reliable information on the reaction rates in the temperature range relevant to this problem.

\subsection{Multicomponent Co-Condensation}

The formation of nanoparticle is described in the framework of the multicomponent co-condensation model (Ref 9,10). The main idea of the model is based on two facts. First, from the phase diagrams of $\mathrm{TaC}$ (Ref 17) or $\mathrm{SiC}$ ( $\mathrm{Ref} 18$ ), it is seen that neither $\mathrm{TaC}$ nor $\mathrm{SiC}$ exist in gaseous state. The compounds are formed after the elements are mixed in liquid droplets. Furthermore, gaseous $\mathrm{Ta}$ and $\mathrm{C}$ (and other combinations of metal and carbon considered below) have strongly different vapor pressures or, in other words, strongly different temperatures of nucleation of liquid droplets at a given partial pressure.

The resulting scenario of particle formation can be summarized as follows. The first step is the homogeneous nucleation of the element with higher condensation temperature, which is Ta in the case of Ta-C system. As soon as stable clusters of this element appear, the remaining atoms of this and another elements start precipitating on the clusters in the process of heterogeneous condensation. The particles collide and coalesce and are transported by the flow. The elements form compounds while being already in liquid form within a particle.

Because of their small size, the droplets solidify at significantly lower temperature than the bulk melting point of the solid (Ref 19). This allows us to assume that the particles stay in the liquid form until their final 
deposition. Furthermore, one can make conclusions about the chemical substance to be formed on the substrate based on the final composition of the particles. The reduction in the melting point also allows us to assume fast coalescence of two particles after their collision.

\subsection{Governing Equations}

The composition of a particle is defined by two internal coordinates $x_{1}$ and $x_{2}$, which denote the numbers of composing atoms (such as Ta and $\mathrm{C}$ ). The distribution of compositions at a space location $\mathbf{r}$ and time moment $t$ is given by the particle density function $n\left(x_{1}, x_{2}, \mathbf{r}, t\right)$. The general dynamic equation for $n$ is (Ref 19)

$$
\begin{aligned}
\frac{\partial n}{\partial t} & +\nabla \cdot(n \mathbf{v})+\frac{\partial}{\partial x_{1}}\left[G_{1} n\right]+\frac{\partial}{\partial x_{2}}\left[G_{2} n\right] \\
= & \nabla \cdot\left(D_{\mathrm{t}} \nabla n\right)+J+\frac{1}{2} \int_{0}^{x_{1}} \int_{0}^{x_{2}} \mathrm{~d} \tilde{x}_{1} \mathrm{~d} \tilde{x}_{2} \beta\left(x_{1}-\tilde{x}_{1}, x_{2}-\tilde{x}_{2}, \tilde{x}_{1}, \tilde{x}_{2}\right) \\
& \times n\left(\tilde{x}_{1}, \tilde{x}_{2}\right) n\left(x_{1}-\tilde{x}_{1}, x_{2}-\tilde{x}_{2}\right) \\
& \left.-\int_{0}^{\infty} \int_{0}^{\infty} \mathrm{d} \tilde{x}_{1} \mathrm{~d} \tilde{x}_{2} \beta\left(x_{1}, x_{2}, \tilde{x}_{1}, \tilde{x}_{2}\right) n\left(x_{1}, x_{2}\right) n\left(\tilde{x}_{1}, \tilde{x}_{2}\right), \quad \text { (Eq } 1\right)
\end{aligned}
$$

where $\nabla=\partial / \partial \mathbf{r}$. According to the equation, the particle distribution changes due to transport by the flow velocity $\mathbf{v}$ (the second term in the left-hand side), growth by heterogeneous condensation of element 1 and element 2 (the third and fourth terms in the left-hand side), turbulent diffusion (the first term in the right-hand side), birth by homogeneous nucleation $J$, and, finally, coagulation of particles due to collisions (the integral terms in the righthand side). Models for the nucleation, condensation, and coagulation terms are presented below. The turbulent diffusivity $D_{\mathrm{t}}$ is defined as $D_{\mathrm{t}}=\mu_{\mathrm{t}} /\left(\rho S c_{\mathrm{t}}\right)$, where $\mu_{\mathrm{t}}$ is the turbulent viscosity calculated by the $k-\varepsilon$ model and $S c_{\mathrm{t}}$ is the Schmidt number taken to be 0.7 .

The evolution of molecular concentrations of gaseous reagents is described by the conservation equations:

$$
\begin{aligned}
\frac{\partial C_{1}}{\partial t}+\nabla \cdot\left(C_{1} \mathbf{v}\right)= & \nabla \cdot\left(\left(D_{1}+D_{\mathrm{t}}\right) \nabla C_{1}\right)-x_{\mathrm{p}}^{*} J \\
& -\int_{0}^{\infty} \int_{0}^{\infty} \mathrm{d} x_{1} \mathrm{~d} x_{2}\left[G_{1} n\right] \\
\frac{\partial C_{2}}{\partial t}+\nabla \cdot\left(C_{2} \mathbf{v}\right)= & \nabla \cdot\left(\left(D_{2}+D_{\mathrm{t}}\right) \nabla C_{2}\right) \\
& -\int_{0}^{\infty} \int_{0}^{\infty} \mathrm{d} x_{1} \mathrm{~d} x_{2}\left[G_{2} n\right] .
\end{aligned}
$$

Here, it is assumed that element 1 is causing the homogeneous nucleation. $x_{\mathrm{p}}^{*}$ is the critical number of atoms in a nucleating particle. $D_{1}$ and $D_{2}$ are the coefficients of molecular diffusion estimated using the Stokes-Einstein expression ( $\operatorname{Ref} 19): D_{i}=k T / f=\frac{3 k T}{2 d_{i}^{2}}[2 \pi p \rho]^{-1 / 2}$, where the friction coefficient $f$ is evaluated using the kinetic energy formula with zero accommodation coefficient, $\rho$ and $p$ are the density and pressure of surrounding argon gas, and $d_{i}$ is the diameter of the reagent gas atom. It is found that the molecular diffusion is comparable with the turbulent diffusion for the reagent gases. The situation is different in the case of nanoparticles, where the molecular diffusion is much smaller than $D_{\mathrm{t}}$ and, thus, can be neglected in (1).

The authors do not include the thermophoresis effect into their first-order model defined by (1) and (2). For the small particles (much smaller than the mean free path), the ratio between the thermodiffusion and the simple diffusion can be reduced to $v / D$, where $v$ is the kinematic viscosity coefficient and $D$ is the diffusion coefficient. The estimations show that this ratio is small even if $D$ is the molecular diffusivity. The ratio turns out to be much less than 1 if the turbulent diffusivity is important. Indeed, the kinematic viscosity of argon at $2000 \mathrm{~K}$ is about $10^{-3} \mathrm{~m}^{2} \mathrm{~s}^{-1}$ (see Fig. 1 and data in Ref 15), while the coefficient of molecular diffusion is of the order of $10^{-2} \mathrm{~m}^{2} \mathrm{~s}^{-1}$ and the coefficient of turbulent diffusion reaches $10^{-1} \mathrm{~m}^{2} \mathrm{~s}^{-1}$ (see Ref 12).

The system of governing equations needs to be complemented by the appropriate conditions on the physical boundaries of the subdomain. It is assumed that concentrations of reagent species are uniformly distributed along the inlet boundary at $r \leq R_{\text {in }}$, where $R_{\text {in }}=0.42 \mathrm{~cm}$ is chosen as a boundary of a zone with sufficiently high temperature so that no nucleation of nanoparticles occurs outside the domain of investigation. Correspondingly, zero concentration of particles at the inlet boundary is assumed. Zero diffusive fluxes of particle density function $n$ and concentrations of chemical species $C_{1}$ and $C_{2}$ are imposed at other physical boundaries. In the internal coordinates, the conditions of zero flux $G_{1} n=0$ and $G_{2} n=0$ at $x_{1}=0$ and $x_{2}=0$, respectively, are applied. The condition $n \rightarrow 0$ at $x_{1}, x_{2} \rightarrow \infty$ is used for evaluation of integrals in (2) and the derivation of approximate model in Sect. 4.4.

\subsection{Models for Homogeneous Nucleation, Heterogeneous Condensation, and Coagulation}

There are numerous theoretical and semiempirical models for the process of homogeneous nucleation. Their common goal is to predict the size of stable nuclei and the nucleation rate given by physical properties and partial pressure of the nucleating gas and ambient temperature. All these theories are based on strong simplifying assumptions and can only give qualitative predictions.

The self-consistent model (Ref 20) is used, according to which the rate of homogeneous nucleation of gas 1 is defined as

$J=2 C_{1} \frac{\sigma^{1 / 2} v_{11}}{\left(2 \pi m_{11}\right)^{1 / 2}} C_{\mathrm{S}, 1} \exp \left(\Theta-\frac{4 \Theta^{3}}{27\left(\ln \left(S_{1}\right)\right)^{2}}\right)$,

where $\Theta=\sigma s_{11} /(k T)$ is the nondimensional coefficient of surface tension. $S_{1}=C_{1} / C_{\mathrm{S}, 1}=p_{1} / p_{\mathrm{S}, 1}$ is the oversaturation level, $p_{1}$ is the partial pressure of the gas, and $C_{\mathrm{S}, 1}$ and $p_{\mathrm{S}, 1}$ are the values of saturation concentration and pressure for this material at the local temperature. $s_{11}, v_{11}$, and $m_{11}$

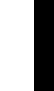


stand for the surface, volume, and mass of one atom of the gas, respectively. The droplets appearing as a result of nucleation are stable if their size, measured by the number of atoms, is larger than the critical size

$$
x_{\mathrm{p}}^{*}=\left(2 \Theta /\left(3 \ln \left(S_{1}\right)\right)\right)^{3} .
$$

The formula (3) is derived under the assumptions that the typical time of nucleation is much smaller than the typical time of the evolution of particles, and that the critical nucleus consists of sufficiently many atoms, generally not fewer than 10. The calculations show that the first assumption is justified in the coating system. On the other hand, the size of a critical nucleus $x_{\mathrm{p}}^{*}$ can become even smaller than the size of one atom. This is the typical result for the nucleation of materials with very low saturation vapor pressure (Ref 19). In the calculations discussed below, all gas molecules of Ta are treated as stable nuclei if $x_{\mathrm{p}}^{*}$ is smaller than 2 .

The rate of the heterogeneous condensation of material $i$ on the stable nucleus is determined by the growth law, for which the kinetic theory expression (Ref 19) is:

$$
G_{i}=\frac{\alpha_{i} p_{\mathrm{S}, i}}{\left(2 \pi m_{1 i} k T\right)^{1 / 2}}\left(S_{i}-1\right) \pi d\left(x_{1}, x_{2}\right)^{2}, \quad i=1,2,
$$

where $d\left(x_{1}, x_{2}\right)$ is the particle diameter and $\alpha_{i}$ is the accommodation coefficient assumed to be equal to 1 .

Frequency of the particle collision and subsequent coagulation can be described by the expression derived from the kinetic theory of gases (Ref 19):

$$
\begin{aligned}
\beta\left(x_{1}, x_{2}, \tilde{x}_{1}, \tilde{x}_{2}\right)= & \left(3 k T / \rho_{\mathrm{p}}\right)^{1 / 2} \times\left(d\left(x_{1}, x_{2}\right)^{-3}+d\left(\tilde{x}_{1}, \tilde{x}_{2}\right)^{-3}\right)^{1 / 2} \\
& \times\left(d\left(x_{1}, x_{2}\right)+d\left(\tilde{x}_{1}, \tilde{x}_{2}\right)\right)^{2} . \quad(\text { Eq 5) }
\end{aligned}
$$

Here $\rho_{\mathrm{p}}$ is the particle density, which, as an approximation, is assumed to be constant equal to the density of final material (e.g., $\mathrm{TaC}$ or $\mathrm{SiC}$ ). It is also assumed that, because of the small size and liquid state of the particles, all collisions are $100 \%$ effective and the coalescence is rapid, so no fractal agglomeration occurs.

\subsection{Method of Solution}

The system of equations and boundary conditions outlined above is useful as a theoretical framework but cannot be solved directly. The main reason is that the adequate resolution of the five-dimensional computational domain is hardly possible at today's level of computational power. Furthermore, even a perfectly accurate numerical solution of the full problem would only present a qualitative picture of the actual process due to the approximate manner in which the key factors, such as thermodynamic and transport coefficients, rate of homogeneous nucleation, growth laws, and the collision frequency function, are determined.

For these reasons, an approximate solution approach conceptually similar to the method of moments is used (Ref 19). The system is reformulated into a system of evolution equations for the statistical parameters of the particle distribution: the number concentration of particles, $N \equiv \int_{0}^{\infty} \int_{0}^{\infty} \mathrm{d} x_{1} \mathrm{~d} x_{2}(n)$, their mean size, $\left(A_{1}+A_{2}\right) / N$, and composition, $A_{2} / A_{1}$. Here, the first moments of the particle density function, $A_{1}=\int_{0}^{\infty} \int_{0}^{\infty} \mathrm{d} x_{1} \mathrm{~d} x_{2}\left(x_{1} n\right)$ and $A_{2}=\int_{0}^{\infty} \int_{0}^{\infty} \mathrm{d} x_{1} \mathrm{~d} x_{2}\left(x_{2} n\right)$, which represent the mean values of the content of elements 1 and 2 in the particles, are introduced. Applying integration in internal coordinates, it is easy to derive

$$
\begin{aligned}
& \frac{\partial N}{\partial t}+\nabla \cdot(N \mathbf{v})=\nabla \cdot\left(D_{\mathrm{t}} \nabla N\right)+J\left(x_{\mathrm{p}}^{*}\right)+J_{\mathrm{col}}, \\
& \frac{\partial A_{1}}{\partial t}+\nabla \cdot\left(A_{1} \mathbf{v}\right)-\int_{0}^{\infty} \int_{0}^{\infty} \mathrm{d} x_{1} \mathrm{~d} x_{2}\left[G_{1} n\right] \\
& =\nabla \cdot\left(\left(D_{1}+D_{\mathrm{t}}\right) \nabla A_{1}\right)+x_{\mathrm{p}}^{*} J\left(x_{\mathrm{p}}^{*}\right), \\
& \frac{\partial A_{2}}{\partial t}+\nabla \cdot\left(A_{2} \mathbf{v}\right)-\int_{0}^{\infty} \int_{0}^{\infty} \mathrm{d} x_{1} \mathrm{~d} x_{2}\left[G_{2} n\right] \\
& =\nabla \cdot\left(\left(D_{2}+D_{\mathrm{t}}\right) \nabla A_{2}\right) .
\end{aligned}
$$

The Eq 6, together with the transport equations for free gas concentrations (2), represent a system for threedimensional (depending on two physical space coordinates and time) fields, which is tractable by standard methods of numerical analysis. One problem remains, though. The term $J_{\text {col }}$ representing the loss of the number of particles due to coagulations and the integrals containing the growth laws, $G_{1}$ and $G_{2}$, cannot be computed without knowledge of the full distribution $n\left(x_{1}, x_{2}, \mathbf{r}, t\right)$ and have to be modeled. This is done in the current work on the basis of the assumption of monodisperse distribution. As discussed in detail in Ref 12, the nucleation rate peaks at physical space locations characterized by strong oversaturation $S_{1}$. This means that on every streamline of the flow, the particles are formed in a localized area and, thus, are characterized by approximately the same initial size $x_{\mathrm{p}}^{*}$. As they follow the streamline, the particles grow in the process of heterogeneous condensation, the rates of which $G_{i}$ are determined by the local flow conditions and the particle size (see (4)). This means that their distribution in internal coordinate space remains nearly monodisperse.

Assuming the monodisperse distribution

$$
\begin{aligned}
n\left(x_{1}, x_{2}, \mathbf{r}, t\right) & \approx N(\mathbf{r}, t) \delta\left(x_{1}-x_{1}^{*}\right) \delta\left(x_{2}-x_{2}^{*}\right), \\
A_{1}(\mathbf{r}, t) & =N x_{1}^{*}, \\
A_{2}(\mathbf{r}, t) & =N x_{2}^{*},
\end{aligned}
$$

it is straightforward to derive the following approximations

$J_{\text {col }}=\frac{1}{2} \beta N^{2}, \quad \beta=\left(3 k T / \rho_{\mathrm{p}}\right)^{1 / 2}\left(\left(A_{1}+A_{2}\right) / N\right)^{1 / 6}$

and

$$
\begin{aligned}
& \int_{0}^{\infty} \int_{0}^{\infty} \mathrm{d} x_{1} \mathrm{~d} x_{2}\left[G_{i} n\right]=N G_{i}^{*}, \\
& G_{i}^{*}=\frac{\alpha_{i} p_{\mathrm{S}, i} \pi\left(S_{i}-1\right)}{\left(2 \pi m_{i} k T\right)^{1 / 2}}\left(\frac{A_{1} d_{i 1}^{3}}{N}+\frac{A_{2} d_{i 2}^{3}}{N}\right)^{2 / 3}, \quad i=1,2,
\end{aligned}
$$

where the numbers of atoms used to determine the volume fractions $d_{i 1}$ and $d_{i 2}$ are given by $x_{1}^{*}$ and $x_{2}^{*}$. 
Two factors neglected in this model tend to widen the peak and destroy the monodisperse character of the distribution and, therefore, should always be taken into careful consideration. The first is the turbulent diffusion (note that the parameters $\left(x_{1}^{*}, x_{2}^{*}\right)$ of the distribution peak vary strongly between the streamlines and, thus, between locations in physical space). This effect can be neglected if the typical residence time of particles within the system is smaller than the typical time of diffusion. As illustrated in Ref 12 , this requirement is satisfied by the current system. The second effect is the coagulation due to random collisions of particles. A detailed discussion of its role in the in-flight synthesis and verification analysis of this approach was provided in Ref 12 . It was shown using a onedimensional one-component model system that the collisions affect the solution during a short period of time immediately after the nucleation. After that, collisions led to rapid decrease of the total number of particles and, correspondingly, to reduction of the collision rate $J_{\text {col }}$ (see (8)). The cumulative impact on the particle distribution parameters $N, A_{1}$, and $A_{2}$ was within the error margin of the other physical models used in this analysis.

The system of Eq 6 with assumptions (7)-(9) was solved numerically in the computation domain shown by the dark gray region in Fig. 1(a) by using the finite-difference approach based on the central-difference scheme of the second order implemented on a uniform rectangular grid. The size of the domain is $\left(Z_{1}-Z_{2}\right) \times R=13 \times 3 \mathrm{~cm}$. The numerical resolution of $450 \times 120$ nodes was found sufficient, with the error of numerical approximation being significantly lower than the errors potentially introduced by the adopted simplifications of the physical model. To use the main flow field for the simulations, the Fluent data for the main gas flow were interpolated onto the new rectangular mesh. The flow data were taken from the computations made on the finest finite-volume mesh with cell size comparable to the step of the finite-difference grid used for the particle simulation.

The last comment concerns verification of the simplified model and numerical approach used in the current study for the process of particle formation. Both were verified in an earlier work (Ref 12) using a posteriori comparison with the direct solution of the full problem (1)-(2) available in the case of one-dimensional onecomponent synthesis. The verification is considered sufficient for the presently studied system since the system retains the principal features that warranted the model validity in Ref 12 .

\section{Results}

\subsection{Synthesis of TaC Nanoparticles}

As mentioned earlier, the analysis of $\mathrm{TaC}$ particle synthesis is conducted for the case of a plasma reactor filled with argon. The formation of nanoparticles is illustrated by Fig. 2 for the inlet concentration of Ta and $\mathrm{C}$ gases equal to $C_{10}=C_{20}=5 \times 10^{21} \mathrm{~m}^{-3}$. Figure 2 contains the evolution plots of the concentrations of gaseous $\mathrm{Ta}$ and $\mathrm{C}$ species, $C_{1}$ (Ta) and $C_{2}$ (C) (Fig. 2a and b); of concentrations of the materials in their condensed form, $A_{1}$ (Ta) and $A_{2}(\mathrm{C})$ (Fig. 2c and d); of the nucleation rate $J$ (Fig. 2e), of the number concentration of particles, $N$ (Fig. 2f); and of the mean diameter and mean composition of $\mathrm{TaC}$ particles (Fig. $2 \mathrm{~g}$ and $\mathrm{h}$ ).

The concentration of atoms of Ta and $\mathrm{C}$ are imposed at the left inlet boundary of the computational domain; the evolution of the free atoms is illustrated in Fig. 2(a) and (b). As temperature of the jet decreases, the partial pressure of Ta vapor overcomes its saturation limit. This starts the process of homogeneous nucleation, which rapidly consumes the free atoms of Ta gas. In the course of the process, the nucleation conditions change rapidly so that soon the critical size $x_{\mathrm{p}}^{*}$ becomes smaller than the size of two Ta atoms. After this moment, all yet free atoms of this material are considered as stable nuclei. The appearance of the nanoparticles marks the initial point of two simultaneous processes. First is the heterogeneous condensation, which is primarily essential for the gas of carbon atoms. The second is the Brownian collisions between the already formed particles.

The temperature of the jet decreases slowly as compared with the results obtained for the plasma jet emitted into the open air atmosphere (Ref 12). As a result, the heterogeneous condensation occurs slowly (see Fig. 3, where the results illustrating the formation of $\mathrm{TaC}$ nanoparticles in the plasma jet injected into the air atmosphere are provided for reference). Based on Fig. 2(b), it can be stated that the processes of homogeneous nucleation and heterogeneous condensation occur entirely within the considered computational domain. However, the particles continue their growth due to collisions at the outlet boundary of the domain, which also leads to a rapid fall in their number (Fig. 2f).

As in Ref 12, one should note that the zone where the nucleation occurs (Fig. 2e) is shifted to the outer boundaries of the jet, which is explained by the effect of strong turbulent diffusion generated in the turbulent jet flow. The radial component of the velocity is very small and cannot explain the observed shift in the nucleation zone. The results reported in Fig. 2 are based on the full velocity field of the main gas flow as obtained in Sect. 3. The results remain the same if the radial velocity is manually taken to be 0 .

The chosen computational domain covers only the initial moment of the particle development; the particles continue to change because of collisions. The effect of collisions is proportional to the square of the particle number concentration and its importance diminishes as the particles move downstream. At the right outlet boundary, the size of the particles is $2.6 \mathrm{~nm}$, which is small, compared with the experimental data (Ref 2). The discrepancy can be explained, firstly, by the fact that particles continue growing, and, secondly, in Ref 2, the particles are collected from the chamber walls or from the gas that leaves the chamber, i.e., after a much longer particle evolution. In addition, the RF plasma gun used for nanoparticle formation in Ref 2 generates less intensive velocity field. The authors of Ref 1,4 , and 11 found that

. 

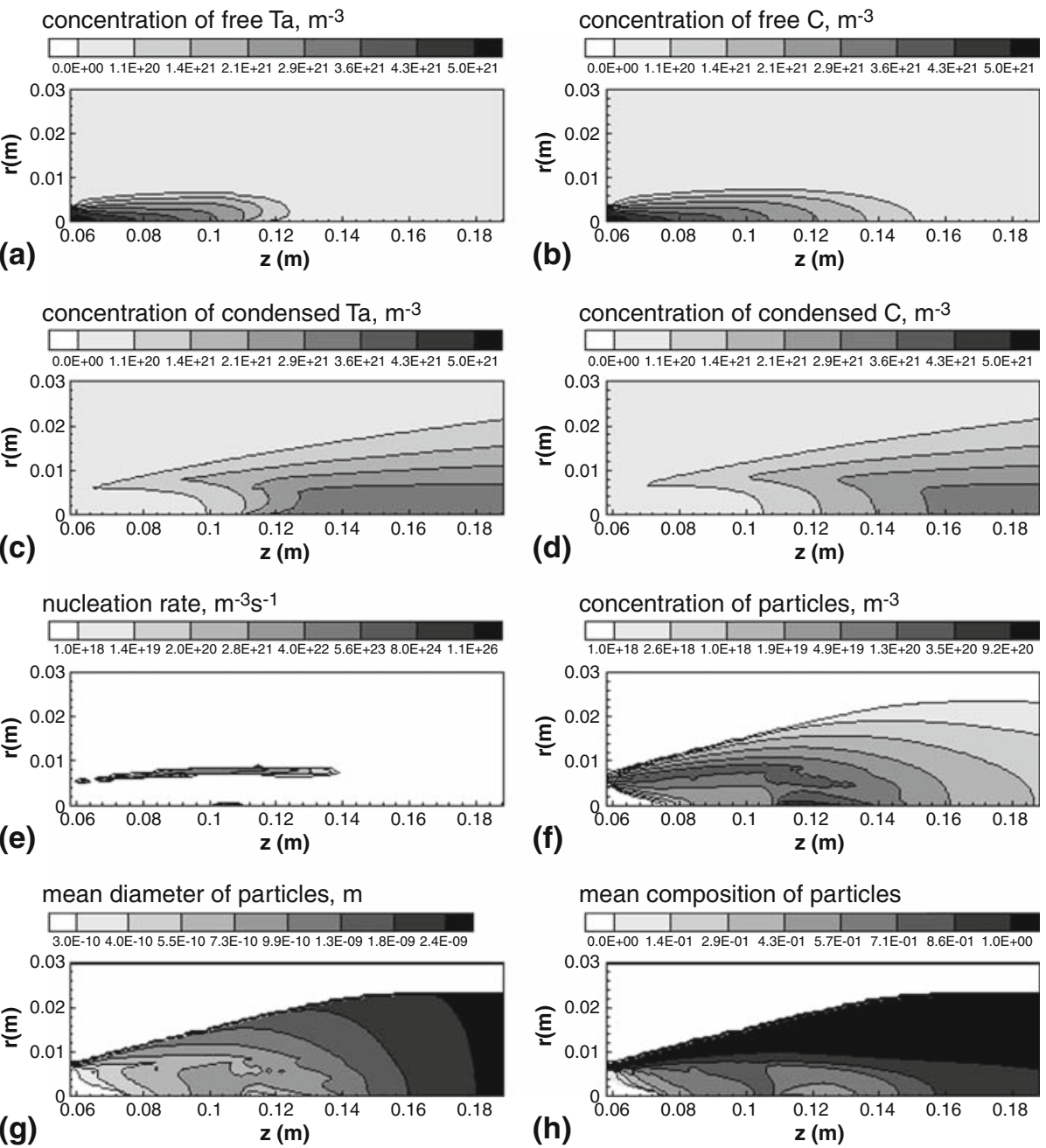

Fig. 2 Formation of $\mathrm{TaC}$ nanoparticles in argon-filled plasma reactor. (a) Concentration of free, not-yet-nucleated, Ta atoms, $C_{1}$, (b) concentration of free $\mathrm{C}$ atoms, $C_{2}$, (c) concentration of the condensed atoms of $\mathrm{Ta}, A_{1}$, (d) concentration of the condensed atoms of $\mathrm{C}$, $A_{2}$, (e) rate of homogeneous nucleation, $J$, (f) concentration of nanoparticles, $N$, (g) mean diameter and (h) mean composition of nanoparticles. The inlet concentrations of Ta and $\mathrm{C}$ vapors are $C_{10}=C_{20}=5 \times 10^{21} \mathrm{~m}^{-3}$. The composition is determined as the ratio of the number of carbon atoms to the number of atoms of metal (Ta) in a particle. The data in plots $(\mathrm{g})$ and (h) are shown for the region with particle concentration $N>10^{18} \mathrm{~m}^{-3}$

the particle size becomes smaller as the cooling rate is increases (by introducing the additional cooling quench to the main jet flow), i.e., the small particle sizes obtained in the present paper could be also explained by the fact that a DC plasma jet has much higher velocity causing higher cooling rate.

The particles at the right outlet boundary have the ratio of Ta to $\mathrm{C}$ atoms equal to 1 (Fig. 2h), similar to what was observed in Ref 12, where the final particle composition was determined by the initial inlet concentration of the Ta and C vapors. This is explained by the complete transition of initially injected atoms of Ta and $\mathrm{C}$ into a condensed state.

\subsection{Other Metal-Carbide Systems}

We also analyzed the in-flight formation of other metalcarbide particles of interest, in particular, $\mathrm{SiC}, \mathrm{TiC}$, and
WC. All these materials can be prepared in similar manner, by using different precursor materials. For instance, $\mathrm{SiC}$ particles can be obtained by injection of the mixture of silicon tetrachloride $\left(\mathrm{SiCl}_{4}\right)$ and methane $\left(\mathrm{CH}_{4}\right)$ into the plasma jet (Ref 3$)$.

The analysis of the saturation vapor pressure (see Appendix) reveals that there is a principal difference in the in-flight nucleation of $\mathrm{TaC}$ and $\mathrm{WC}$ particles from the synthesis of $\mathrm{SiC}$ and TiC. Formation of the WC particles follows the scenario discussed in the preceding section, and can be illustrated by the set of plots totally similar to Fig. 2.

In the case of $\mathrm{SiC}$ and $\mathrm{TiC}$ systems, the nucleation occurs differently, as in this case metal ( $\mathrm{Si}$ and $\mathrm{Ti}$ ) possesses lower saturation vapor pressure. For such systems, the nucleation should start with formation of carbon nanoparticles, with the metal precipitating on the surface 

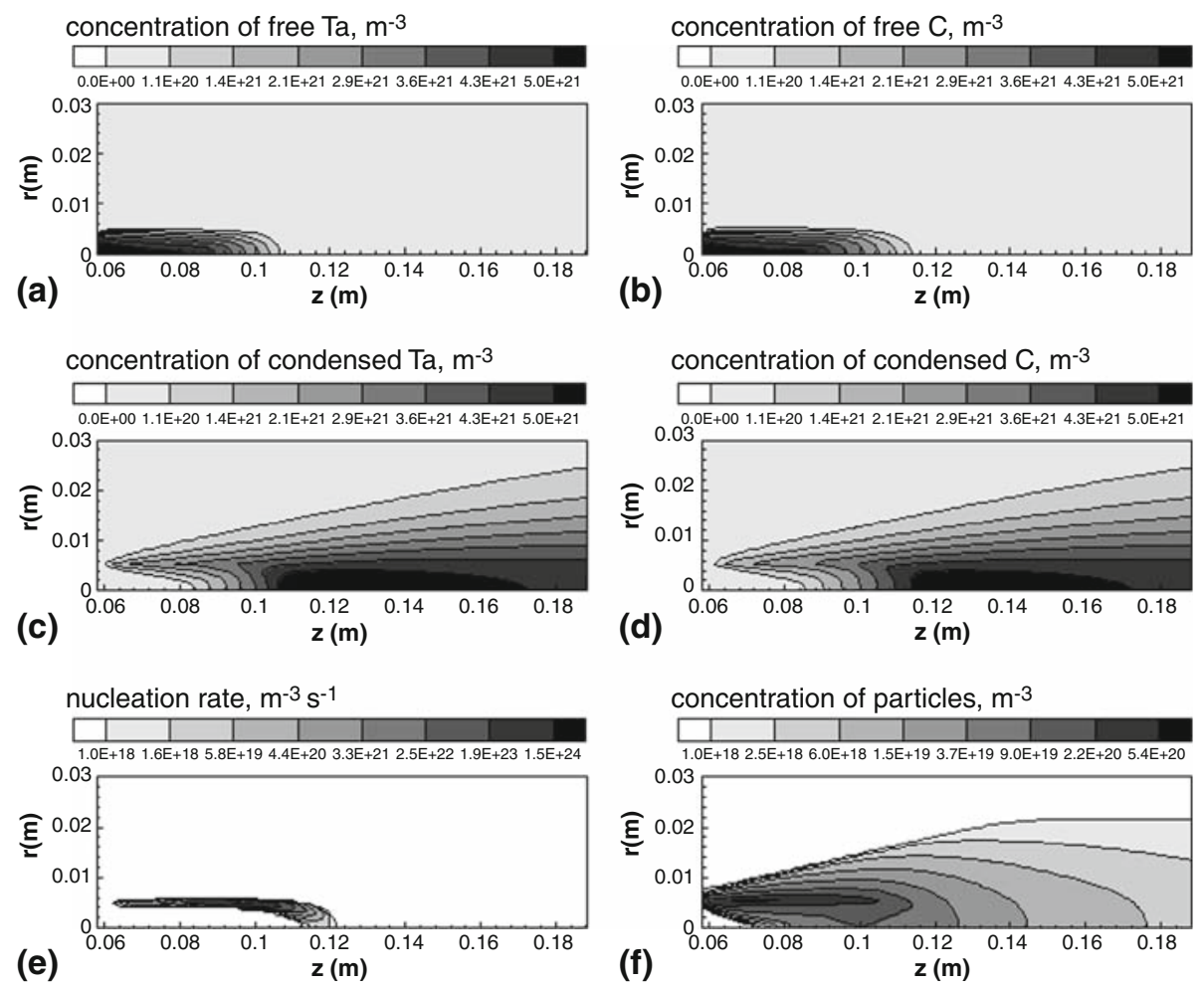

Fig. 3 Formation of TaC nanoparticles in an open air atmosphere. (a) Concentration of free Ta atoms, $C_{1}$, (b) concentration of free $\mathrm{C}$ atoms, $C_{2}$, (c) concentration of the condensed atoms of Ta, $A_{1}$, (d) concentration of condensed atoms of C, $A_{2}$, (e) rate of homogeneous nucleation, $J$, (f) concentration of nanoparticles, $N$. The inlet concentrations of Ta and C vapors are $C_{10}=C_{20}=5 \times 10^{21} \mathrm{~m}^{-3}$

of the nuclei. The analysis becomes more complicated, taking into account the fact that carbon does not exist in the liquid state, at least in bulk volumes, under atmospheric pressure (Ref 21). The simulations of the preceding section can be accomplished by using the free surface energy for a solid material instead of the surface tension.

The results of numerical simulations fulfilled for the Si-C system are summarized in Fig. 4. The synthesis of $\mathrm{TiC}$ nanoparticles would occur very similar to this case. Figure 4 is organized in complete correspondence to Fig. 2 and contains the set of plots obtained for the equal inlet concentrations of $\mathrm{C}$ and $\mathrm{Si}, C_{10}=C_{20}=5 \times 10^{21} \mathrm{~m}^{-3}$. In Fig. 4(h), the particle composition is defined as the ratio of number of carbon atoms to the number of atoms of silicon. At the initial moment the particles are made up of carbon, i.e., the composition ratio is infinite. Later on, the mean particle composition decreases and approaches 1 .

The analysis of formation of $\mathrm{SiC}$ and $\mathrm{TiC}$ nanoparticles shows that lower saturation pressures of nucleating gases delay the processes of homogeneous nucleation and heterogeneous condensation. The condensation of free atoms of metal remains nonnegligible even at the right outlet border of the computational domain. Even at this border, not all atoms of Si gas, injected into the plasma reactor, are condensed (Fig. 4b). This also explains the variation in the mean particle composition (from 1.3 at the symmetry axis to 1 at the outer regions) along the right outlet boundary of the considered domain.

\section{Conclusions}

The model for the in-flight synthesis of the twocomponent nanoparticles developed in Ref 12 can be easily adapted for the analysis of the nucleation of various compounds from different precursors that are injected into the thermal plasma jet. The model is based on several simplifying assumptions and can only be used for the qualitative description of the nucleation and growth processes. A more quantitative approach is hindered by the lack of understanding and reliable information concerning the essential physical properties of various processes on the nanoscale. This, in particular, concerns the process of nucleation subject to very high supersaturation levels, when the nucleus size reaches the size of an atom. In the current work, the authors extrapolate the classical approach on this process.

Albeit of approximate nature, this model produces interesting and qualitatively adequate results regarding the in-flight synthesis of metal-carbide nanoparticles. The model predicts the essential role of turbulence on the nucleating dynamics. The turbulent nature of the highvelocity jet shifts the zone where the nucleation takes place and has effect on the size of nuclei. In addition, the co-condensation model allows us to consider how the particle composition varies during the in-flight synthesis. This helps to correlate the amount of the precursor used and the size 

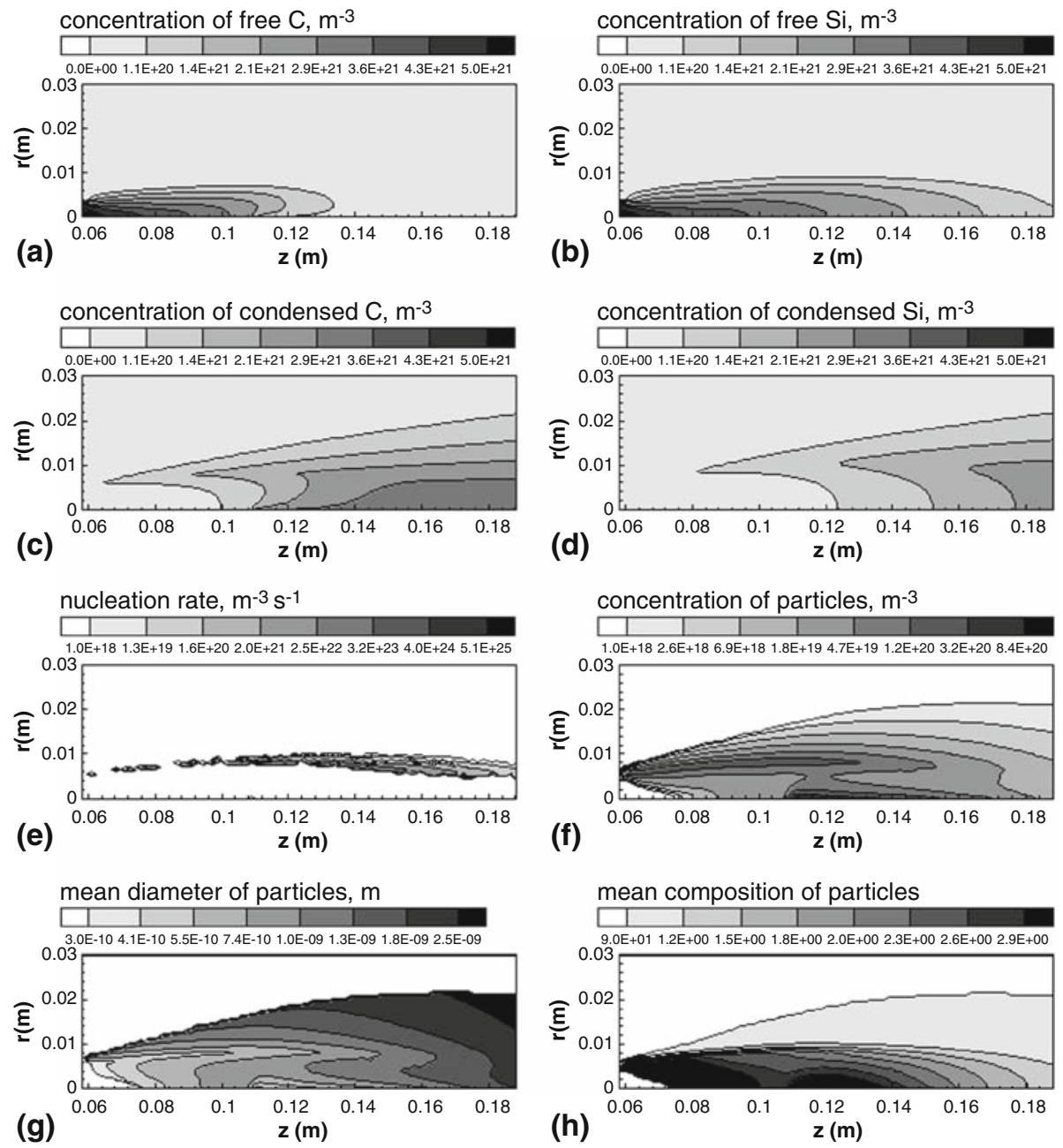

Fig. 4 Formation of $\mathrm{SiC}$ nanoparticles in an argon-filled plasma reactor. (a) Concentration of free $\mathrm{C}$ atoms, $C_{1}$, (b) concentration of free $\mathrm{Si}$ atoms, $C_{2}$, (c) concentration of the condensed atoms of $\mathrm{C}, A_{1}$, (d) concentration of the condensed atoms of $\mathrm{Si}, A_{2}$, (e) rate of homogeneous nucleation, $J$, (f) concentration of nanoparticles, $N$, (g) mean diameter, and (h) mean composition of nanoparticles. The inlet concentrations of $\mathrm{C}$ and $\mathrm{Si}$ vapors are $C_{10}=C_{20}=5 \times 10^{21} \mathrm{~m}^{-3}$. The composition is determined as the ratio of the number of carbon atoms to the number of atoms of metal $(\mathrm{Si})$ in a particle. The data in plots $(\mathrm{g})$ and $(\mathrm{h})$ are shown for the region with particle concentration $N>10^{18} \mathrm{~m}^{-3}$

and composition of the nanoparticles, as well as the preferable zone where the nanoparticles should be collected.

One of the most important conclusions is underlining of the principal differences in the nucleation scenarios between $\mathrm{TaC}$ and $\mathrm{WC}$ systems from $\mathrm{SiC}$ and $\mathrm{TiC}$. The co-condensation approach is based on the idea that formation of the nanoparticles begins with nucleation of the element that has lower vapor pressure. For Ta-C and $\mathrm{W}-\mathrm{C}$ systems, metal initiates the formation of particles. The synthesis of $\mathrm{SiC}$ and $\mathrm{TiC}$ nanoparticles starts with sublimation of carbon. The analysis becomes even more complex taking into account the fact that the classical theories of the homogeneous nucleation presuppose that the vapor nucleates into liquid state, which does not happen for carbon as carbon does not exist in liquid state under atmospheric pressure.

The theoretical predictions of the co-condensation theory could be experimentally verified by collecting the nanoparticles at different distances from the plasma gun's outlet. For the system of the first kind (TaC and WC), the particles should have smaller carbon content at short distances, and the carbon content should increase downstream; the particles of the second kind ( $\mathrm{SiC}$ and $\mathrm{TiC}$ ) should be carbon-rich at shorter distances and the carbon content would decrease downstream.

\section{Acknowledgments}

The authors are thankful to the reviewers for insightful and constructive comments.

\section{Appendix}

\subsection{Physical Properties}

The referenced points for $\mathrm{Ta}, \mathrm{C}$, and $\mathrm{Si}$ gases used for the simulations are summarized in Table 1. Between the 
Table 1 Vapor pressure reference points

\begin{tabular}{lccccccr}
\hline $\boldsymbol{P}, \mathbf{P a}$ & $\mathbf{1}$ & $\mathbf{1 0}$ & $\mathbf{1 0}^{\mathbf{2}}$ & $\mathbf{1 0}^{\mathbf{3}}$ & $\mathbf{1 0}^{\mathbf{4}}$ & $\mathbf{1 0}^{\mathbf{5}}$ & $\boldsymbol{p}_{\text {atm }}$ \\
\hline Carbon: $T, \mathrm{~K}$ & - & 2839 & 3048 & 3289 & 3572 & 3908 & - \\
Tantalum: T, K & 3297 & 3597 & 3957 & 4395 & 4939 & 5634 & 5731 \\
Tungsten: $T, \mathrm{~K}$ & 3477 & 3773 & 4137 & 4579 & 5127 & 5823 & 5828 \\
Silicon: $T, \mathrm{~K}$ & 1908 & 2102 & 2339 & 2636 & 3021 & 3537 & 3538 \\
Titanium: T, K & 1982 & 2171 & 2403 & 2692 & 3064 & 3558 & 3560
\end{tabular}

The data are taken from Ref 22 and 23

data points, the vapor pressure was approximated by the Clausius-Clapeyron equation:

$$
p=p_{\text {ref }} \exp \left(-\frac{Q}{R}\left(\frac{1}{T}-\frac{1}{T_{\text {ref }}}\right)\right) .
$$

Here $p_{\text {ref }}$ and $T_{\text {ref }}$ are the values given in Table $1, Q$ is the latent heat of vaporization per mole, and $R$ is the universal gas constant. For the latent heat of vaporization the following values were used: for Ta, $Q=3.65 \times 10^{5} \mathrm{~J}$ $\mathrm{mol}^{-1}(\operatorname{Ref} 24)$, for C, $Q=3.56 \times 10^{5} \mathrm{~J} \mathrm{~mol}^{-1}(\operatorname{Ref} 25)$, and for Si, $Q=3.59 \times 10^{5} \mathrm{~J} \mathrm{~mol}^{-1}$ (Ref 24).

For the homogeneous nucleation of Ta gas, the value of the surface tension coefficient $\sigma=2.07 \mathrm{~N} \mathrm{~m}^{-1}$, reported in Ref 26, was used. In the calculations in the current study, the temperature variations exceed the reported measurement interval. The authors chose to extrapolate the constant value for the surface tension coefficient on the entire temperature range where the particles exist. Following the common assumption of the classical nucleation theories the dependence of the surface tension on the particle size is not considered. To describe the process of nucleation of $\mathrm{Si}-\mathrm{C}$, the surface tension of $C$ is needed. No measurements of the surface tension for carbon were found in the literature, which is likely because of the fact that carbon is not observed in a liquid form under atmospheric pressure (Ref 21). To estimate the value of the surface tension, the results of measurements of the free surface energy of solid carbon fibers (Ref 27), $\sigma=0.046 \mathrm{~J} \mathrm{~m}^{-2}$, were used.

The density of $\mathrm{TaC}$ in solid form is $1.39 \times 10^{4} \mathrm{~kg} \mathrm{~m}^{-3}$, and the density of $\mathrm{SiC}$ is $3.22 \times 10^{3} \mathrm{~kg} \mathrm{~m}^{-3}$. To calculate the size of particles, the following diameters of Ta and $\mathrm{C}$ atoms were assumed, $2.7 \times 10^{-10} \mathrm{~m}$ and $2.6 \times 10^{-10} \mathrm{~m}$; the diameter of Si atoms was taken as $2.2 \times 10^{-10} \mathrm{~m}$.

\section{References}

1. B.M. Goortani, P. Proulx, S. Xue, and N.Y. Mendoza-Gonzalez, Controlling Nanostructure in Thermal Plasma Processing: Moving from Highly Aggregated Porous Structure to Spherical Silica Nanoparticles, Powder Technol., 2007, 175, p 22-32

2. T. Ishigaki, S.-M. Oh, J.-G. Li, and D.-W. Park, Controlling the Synthesis of $\mathrm{TaC}$ Nanopowders by Injecting Liquid Precursor into RF Induction Plasma, Sci. Technol. Adv. Mater., 2005, 6, p 111-118

3. S.-M. Oh, M. Cappelli, and D.-W. Park, Preparation of NanoSized Silicon Carbide Powder Using Thermal Plasma, Korean J. Chem. Eng., 2002, 19(5), p 903-907

4. R. Ye, J.-G. Li, and T. Ishigaki, Controlled Synthesis of Alumina Nanoparticles Using Inductively Coupled Thermal Plasma with Enhanced Quenching, Thin Solid Films, 2007, 515, p 4251-4257
5. M. Desilets, J.-F. Bilodeau, and P. Proulx, Modelling of the Refractive Synthesis of Ultra-Fine Powders in a Thermal Plasma Reactor, J. Phys. D: Appl. Phys., 1997, 30, p 1951-1960

6. J.S. McFeaters, R.L. Stephens, P. Schwerdtfeger, and M. Liddell, Numerical Modeling of Titanium Carbide Synthesis in Thermal Plasma Reactors, Plasma Chem. Plasma Proc., 1994, 14(3), p 333360

7. N.Y. Mendoza-Gonzalez, B.M. Goortani, and P. Proulx, Numerical Simulation of Silica Nanoparticles Production in an RF Plasma Reactor: Effect of Quench, Mater. Sci. Eng. C, 2007, 27(5-8), p 1265-1269

8. A.B. Murphy, Formation of Titanium Nanoparticles from a Titanium Tetrachloride Plasma, J. Phys. D: Appl. Phys., 2004, 37, p 2841-2847

9. M. Shigeta and T. Watanabe, Multi-Component Co-Condensation Model of Ti-Based Boride/Silicide Nanoparticle Growth in Induction Thermal Plasmas, Thin Solid Films, 2007, 515, p 4217-4250

10. M. Shigeta and T. Watanabe, Growth Mechanism of SiliconBased Functional Nanoparticles Fabricated by Inductively Coupled Thermal Plasmas, J. Phys. D: Appl. Phys., 2007, 40, p 2407-2419

11. M. Shigeta and T. Watanabe, Numerical Investigation of Cooling Effect on Platinum Nanoparticle formation in Inductively Coupled Thermal Plasmas, J. Appl. Phys., 2008, 103, p 074903

12. A. Vorobev, O. Zikanov, and P. Mohanty, Modelling of the In-Flight Synthesis of TaC Nanoparticles from Liquid Precursor in Thermal Plasma Jet, J. Phys. D: Appl. Phys., 2008, 41, 085302 $(15 \mathrm{p})$

13. J.P. Trelles, E. Pfender, and J. Heberlein, Multiscale Finite Element Modeling of Arc Dynamics in a DC Plasma Torch, Plasma Chem. Plasma Proc., 2006, 26(6), p 557-575

14. T.-H. Shih, W.W. Liou, A. Shabbir, Z. Yang, and J. Zhu, A New k- $\varepsilon$ Eddy Viscosity Model for High Reynolds Number Turbulent Flows, Comput. Fluids, 1995, 24(3), p 227-238

15. W.L.T. Chen, J. Heberlein, and E. Pfender, Critical Analysis of Viscosity Data of Thermal Argon Plasmas at Atmospheric Pressure, Plasma Chem. Plasma Proc., 1996, 16(4), p 635-650

16. B. Liu, T. Zhang, and D.T. Gawne, Computational Analysis of the Influence of Process Parameters on the Flow Field of a Plasma Jet, Surf. Coat. Technol., 2000, 132, p 202-216

17. A Critical Review of Refractories, Los Alamos Scientific Laboratory of the University of California, Los Alamos, NM, 1964

18. Yu.M. Tairov and V.F. Tsvetkov, Semiconductor Compounds $\mathrm{A}^{\mathrm{IV}} \mathrm{B}^{\mathrm{IV}}$, Handbook on Electrotechnical Materials, Vol. 3, Yu.V. Koritskii, V.V. Pasynkov, and B.M. Tareev, Ed., Energomashizdat, Leningrad, 1988, p 446-471 (in Russian)

19. S.K. Friedlander, Smoke, Dust, and Haze. Fundamentals of Aerosol Dynamics, Oxford University Press, 2000

20. S.L. Girshick, C.-P. Chiu, and P.H. McMurry, Time-Dependent Aerosols Models and Homogeneous Nucleation Rates, Aerosol Sci. Technol., 1990, 13, p 465-477

21. J. Zazula, On Graphite Transformations at High Temperature and Pressure Induced by Absorption of the LHC Beam, CERN-LHCProject-Note-78, 1997

22. H.R. Leider, O.H. Krikorian, and D.A. Young, Thermodynamic Properties of Carbon up to the Critical Point, Carbon, 1973, 11(5), p 555-563

23. D. Stull, American Institute of Physics Handbook, 3rd ed., D.E. Gray, Ed., McGraw-Hill, New York, 1972

24. J.A. Dean, Lange's Handbook of Chemistry, 15th ed., McGrawHill, New York, 1999

25. R.H. Schumm, D.D. Wagman, S. Bailey, W.H. Evans, and V.B. Parker, National Bureau of Standards (USA), Technical Notes $270-1$ to $270-8,1973$

26. A.P. Miiller and A. Cezairliyan, Measurement of Surface Tension of Tantalum by a Dynamic Technique in a Microgravity Environment, Int. J. Thermophys., 1993, 14(5), p 1063-1075

27. S. Nuriel, L. Liu, A.H. Barber, and H.D. Wagner, Direct Measurement of Multiwall Nanotube Surface Tension, Chem. Phys. Lett., 2005, 404, p 263-266 